\title{
A GENERALIZATION OF EVANS' THEOREM: EMBEDDING PARTIAL TRICYCLE SYSTEMS
}

\author{
C. C. LINDNER and C. A. RODGER
}

(Received 2 April 1993; revised 4 May 1995)

\author{
Communicated by L. Caccetta
}

\section{Dedicated to the memory of Trevor Evans}

\begin{abstract}
In 1960, Trevor Evans gave a best possible embedding of a partial latin square of order $n$ in a latin square of order $t$, for any $t \geq 2 n$. A latin square of order $n$ is equivalent to a 3-cycle system of $K_{n, n, n}$, the complete tripartite graph. Here we consider a small embedding of partial $3 k$-cycle systems of $K_{n, n, n}$ of a certain type which generalizes Evans' Theorem, and discuss how this relates to the embedding of patterned holes, another recent generalization of Evans' Theorem.
\end{abstract}

1991 Mathematics subject classification (Amer. Math. Soc.): 05B15.

\section{Introduction}

In what follows, to say that the complete undirected tripartite graph $K_{n, n, n}$ is based on $Q \times\{1,2,3\}$ will always mean that the tripartition of the vertices is: $Q \times\{1\}, Q \times\{2\}$ and $Q \times\{3\}$. A $3 k$-tricycle of $K_{n, n, n}$ is a cycle of length $3 k$ of the form depicted in Figure 1 , which we will denote by any cyclic shift of $\left(\left(x_{1}, 1\right),\left(y_{1}, 2\right),\left(z_{1}, 3\right),\left(x_{2}, 1\right), \ldots\right.$, $\left.\left(z_{k}, 3\right)\right)$ or $\left(\left(y_{1}, 2\right),\left(x_{1}, 1\right),\left(z_{k}, 3\right),\left(y_{k}, 2\right), \ldots,\left(z_{1}, 3\right)\right)$. The definition implies that the $k$ elements in each of the sets $\left\{x_{1}, x_{2}, x_{3}, \ldots, x_{k}\right\},\left\{y_{1}, y_{2}, y_{3}, \ldots, y_{k}\right\}$ and $\left\{z_{1}, z_{2}, z_{3}, \ldots, z_{k}\right\}$ are distinct.

A $3 k$-tricycle system $(3 k T S)$ of order $n$ is a pair $(Q \times\{1,2,3\}, T)$, where $T$ is a collection of edge disjoint $3 k$-tricycles which partition $K_{n, n, n}$ based on $Q \times\{1,2,3\}$.

It is well-known that a 3-tricycle system $(Q \times\{1,2,3\}, T)$ of order $n$ is equivalent to a quasigroup $(Q, \circ)$; the equivalence being $((a, 1),(b, 2),(c, 3)) \in T$ if and only

First and second authors supported respectively by NSF grants DMS-8913576 and DMS-9024645.

(C) 1995 Australian Mathematical Society 0263-6115/95 \$A2.00+0.00 


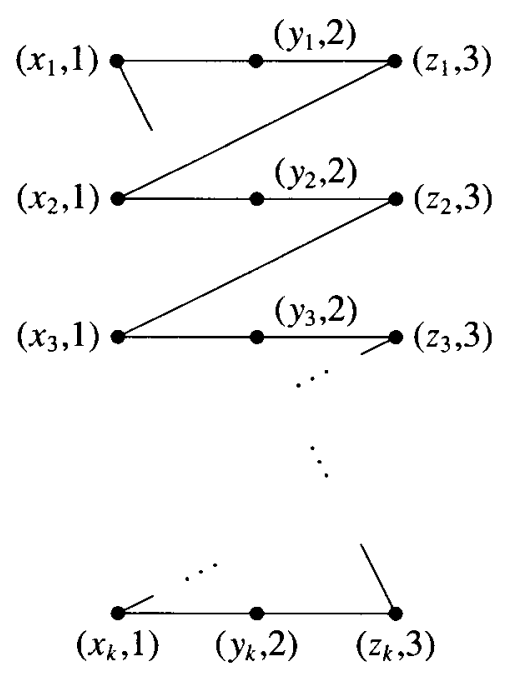

FiguRE 1. The tricycle $\left(\left(x_{1}, 1\right),\left(y_{1}, 2\right),\left(z_{1}, 3\right),\left(x_{2}, 1\right), \ldots,\left(z_{k}, 3\right)\right)$

if $a \circ b=c$. Beyond this, not a lot is known.

EXAMPLE 1.1. (3-tricycle system of order 4.)

$$
\begin{aligned}
T= & \{(11,12,13),(31,12,23),(11,22,33),(31,22,43),(11,32,43),(31,32,33), \\
& (11,42,23),(31,42,13),(21,12,43),(41,12,33),(21,22,23),(41,22,13), \\
& (21,32,13),(41,32,23),(21,42,33),(41,42,43)\}
\end{aligned}
$$

\begin{tabular}{|c|c|c|c|c|}
\hline 0 & 1 & 2 & 3 & 4 \\
\hline 1 & 1 & 3 & 4 & 2 \\
\hline 2 & 4 & 2 & 1 & 3 \\
\hline 3 & 2 & 4 & 3 & 1 \\
\hline 4 & 3 & 1 & 2 & 4 \\
\hline
\end{tabular}

equivalent quasigroup 
EXAMPLE 1.2. (6-tricycle system of order 4.)

$$
\begin{aligned}
T= & (11,12,13,21,22,23),(31,32,33,41,42,43),(11,22,33,21,32,13), \\
& (11,32,43,21,42,33),(11,42,23,21,12,43),(31,12,23,41,22,13), \\
& (31,22,43,41,32,23),(31,42,13,41,12,33)\}
\end{aligned}
$$

A partial $3 k T S$ of order $n$ is a pair $(Q \times\{1,2,3\}, P)$, where $P$ is a collection of edge disjoint $3 k$-tricycles of $K_{n, n, n}$. The difference between a partial $3 k T S$ and a $3 k T S$ is that the $3 k$-tricycles of a partial $3 k T S$ do not necessarily partition $K_{n, n, n}$.

Now given a partial $3 k T S(Q \times\{1,2,3\}, P)$ of order $n$ there is the obvious problem of completing $(Q \times\{1,2,3\}, P)$ to a $3 k T S$. That is to say, partitioning $K_{n, n, n} \backslash P$ (the complement of $P$ in $\left.K_{n, n, n}\right)$ into edge disjoint $3 k$-tricycles. The following example shows that in general this cannot be done.

EXAMPLE 1.3. (Partial $6 T S$ of order 3.) Let $Q=\{1,2,3\}$. Then $(Q \times\{1,2,3\}, P)$ where $P=\{(11,12,13,21,22,23),(11,22,33,21,32,13)\}$ is a partial $6 T S$ of order 3. Since $\left|E\left(K_{3,3,3} \backslash P\right)\right|=15$, no amount of effort will partition $K_{3,3,3} \backslash P$ into edge disjoint 6-tricycles.

Since a partial $3 k T S$ cannot necessarily be completed, the problem of embedding is immediate. The (partial) $3 k T S(Q \times\{1,2,3\}, P)$ is said to be embedded in the $3 k T S$ $\left(Q^{*} \times\{1,2,3\}, P^{*}\right)$ provided $Q \subseteq Q^{*}$ and $P \subseteq P^{*}$. Naturally, if an embedding is possible we would like $\left|Q^{*}\right|$ to be as small as possible. A quick check shows that the partial $6 T S$ of order 3 in Example 1.3 is embedded in the $6 T S$ of order 4 in Example 1.2 (and you can't get much smaller than that!)

In 1960 Trevor Evans [1] proved that a partial quasigroup of order $n$ can always be embedded in a quasigroup of order $t$ for every $t \geq 2 n$. This is the best possible result of its kind since it is always possible to construct a partial quasigroup of order $n$ which cannot be embedded in a quasigroup of order less than $2 n$ for every $n \geq 4$. Since a (partial) $3 T S$ is equivalent to a quasigroup, the problem of embedding a partial $3 T S$ is completely solved.

However, the problem of embedding a partial $3 k T S$ for $3 k \geq 6$ is an open problem. The object of this paper is to use a recent generalization [2,3] of Evans' Theorem on embedding partial quasigroups (to groupoids), to not only obtain an embedding of a general partial $3 k T S$, but a fairly 'small' embedding as well. In particular, we prove that when $3 k \notin\{6,18\}$, a partial $3 k T S$ of order $n$ can be embedded in a $3 k T S$ of order $k t$ for every $t \geq 2 n$, and of order $2 k t$ when $3 k \in\{6,18\}$. As mentioned above, this is the best possible result for $k=1$. For $k>1$ this is undoubtedly not the best possible embedding, but is still pretty good. At this stage it is not exactly clear what the best possible embedding is. A lot of work remains to be done on this problem. 


\section{Embedding groupoids}

In order to achieve the embedding results in this paper we will need the following generalization of Evans' Theorem.

A pair of partial groupoids $\left(Q, \circ_{1}\right)$ and $\left(Q, \circ_{2}\right)$ is called an embedding pair of partial groupoids provided that:

(1) exactly the same products are defined in $\left(Q, \circ_{1}\right)$ and $\left(Q, \circ_{2}\right)$,

(2) each element $x \in Q$ occurs as a product the same number of times in $\left(Q, \circ_{1}\right)$ and $\left(Q, \circ_{2}\right)$,

(3) $\left(Q, o_{1}\right)$ is row latin, and

(4) $\left(Q, O_{2}\right)$ is column latin.

EXAMPLE 2.1. (An embedding pair of groupoids $\left(Q, \circ_{1}\right)$ and $\left(Q, \circ_{2}\right)$ of order 6.$)$

\begin{tabular}{c|c|c|c|c|c|c|}
$o_{1}$ & 1 & 2 & 3 & 4 & 5 & 6 \\
\hline 1 & & 2 & 5 & & & \\
\cline { 2 - 7 } 2 & & & & 6 & & 4 \\
\cline { 2 - 7 } 3 & 1 & & 5 & & & \\
\cline { 2 - 7 } 4 & & 2 & & & 3 & \\
\cline { 2 - 7 } 5 & & & & & & \\
\cline { 2 - 7 } 6 & & 6 & & & & 2 \\
\cline { 2 - 7 } & & & & &
\end{tabular}

\begin{tabular}{|c|c|c|c|c|c|c|}
\hline $\mathrm{O}_{2}$ & 1 & 2 & 3 & 4 & 5 & 6 \\
\hline 1 & & 2 & 2 & & & \\
\hline 2 & & & & 3 & & 4 \\
\hline 3 & 5 & & 5 & & & \\
\hline 4 & & 1 & & & 6 & \\
\hline 5 & & & & & & \\
\hline 6 & & 6 & & & & 2 \\
\hline
\end{tabular}

Now let $(P, \circ)$ be a partial quasigroup with the undefined products being precisely the products which are defined in $\left(Q, \circ_{1}\right)$ and $\left(Q, \circ_{2}\right)$. Denote by $\left(P, \circ\left(\circ_{1}\right)\right)$ the groupoid obtained from $(P, O)$ by defining the undefined products with the products from $\left(Q, \circ_{1}\right)$, and by $\left(P, \circ\left(\circ_{2}\right)\right)$ the groupoid obtained from $(P, \circ)$ by defining the undefined products with the products from $\left(Q, \circ_{2}\right)$. If $\left(P, \circ\left(\circ_{1}\right)\right)$ is row latin and $\left(P, \circ\left(\circ_{2}\right)\right)$ is column latin we will say that the embedding pair of partial groupoids is embedded in the partial quasigroup $(P, \circ)$. In this case the ordered triple $\left(\left(Q, \circ_{1}\right),\left(Q, \circ_{2}\right),(P, \circ)\right)$ is known as a patterned hole [2].

EXAMPLE 2.2. The embedding pair of partial groupoids of order 6 in Example 2.1 is embedded in the following partial quasigroup $(P, \circ)$ of order 9 . Check it out! 


\begin{tabular}{c|c|c|c|c|c|c|c|c|c|}
$\circ$ & \multicolumn{1}{|c|}{1} & 2 & 3 & 4 & 5 & 6 & 7 & 8 & 9 \\
\hline 1 & 1 & & & 4 & 7 & 8 & 9 & 3 & 6 \\
\cline { 2 - 10 } 2 & 7 & 8 & 9 & & 5 & & 3 & 2 & 1 \\
\cline { 2 - 10 } 3 & & 4 & & 9 & 2 & 3 & 6 & 7 & 8 \\
\cline { 2 - 10 } 4 & 6 & & 7 & 5 & & 1 & 4 & 8 & 9 \\
\cline { 2 - 10 } 5 & 2 & 5 & 1 & 6 & 8 & 9 & 7 & 4 & 3 \\
\cline { 2 - 10 } 6 & 4 & & 3 & 1 & 9 & & 8 & 5 & 7 \\
\cline { 2 - 10 } 7 & 9 & 7 & 4 & 8 & 3 & 5 & 1 & 6 & 2 \\
\cline { 2 - 10 } 8 & 8 & 3 & 6 & 2 & 1 & 7 & 5 & 9 & 4 \\
\cline { 2 - 10 } 9 & 3 & 9 & 8 & 7 & 4 & 6 & 2 & 1 & 5 \\
\cline { 2 - 9 } & & & & & & & &
\end{tabular}

$(P, \circ)$

THEOREM 2.3. (C. C. Lindner and C. A. Rodger [2].) An embedding pair of partial groupoids of order $n$ can be embedded in a partial quasigroup of order $t$ for every $t \geq 2 n$.

We remark that, as with Evans' Theorem, this is the best possible result with respect to the size of the containing partial quasigroup.

With Theorem 2.3 in hand we can now proceed to the embedding of a partial $3 k T S$.

\section{Embedding a $3 k T S$}

For technical reasons (which will become abundantly clear) we break the construction into two parts: all $3 k \neq 6$ or 18 and $3 k \in\{6,18\}$.

First case: $3 \mathrm{k} \neq 6$ or 18 . Let $(Q \times\{1,2,3\}, P)$ be a partial $3 k T S$ of order $n$, where $3 k \neq 6$ or 18 . Define a pair of partial groupoids as follows: For each $3 k$-tricycle $\left(\left(x_{1}, 1\right),\left(y_{1}, 2\right),\left(z_{1}, 3\right), \ldots,\left(z_{k}, 3\right)\right)$ belonging to $P$ define:

$$
\left\{\begin{array}{ccc|cc}
x_{1} \circ_{1} & y_{1}=z_{k} & x_{1} \circ_{2} & y_{1}=z_{1} \\
x_{2} & \circ_{1} & y_{2}=z_{1} & x_{2} \circ_{2} & y_{2}=z_{2} \\
\vdots & \vdots & \vdots \\
x_{k} \circ_{1} & y_{k}=z_{k-1} & x_{k} \circ_{2} & y_{k}=z_{k}
\end{array}\right.
$$

It is straightforward and not difficult to show that $\left(Q, \circ_{1}\right)$ and $\left(Q, o_{2}\right)$ is an embedding pair of partial groupoids of order $n$. Let $\left(Q^{*}, \circ\right)$ be a partial quasigroup of order $t \geq 2 n$ 
in which $\left(Q, \circ_{1}\right)$ and $\left(Q, \circ_{2}\right)$ can be embedded. Finally, let $(K, \otimes)$ be an idempotent quasigroup of order $k$ having an orthogonal mate. (Since $k \neq 2$ or $6,(K, \otimes)$ exists.) We can partition $(K, \otimes)$ into $k$ disjoint transversals

$$
\pi_{i}=\left(\begin{array}{ccccc}
x_{i 1}=1 & x_{i 2}=2 & x_{i 3}=3 & \ldots & x_{i k}=k \\
y_{i 1} & y_{i 2} & y_{i 3} & \ldots & y_{i k} \\
z_{i 1} & z_{i 2} & z_{i 3} & \ldots & z_{i k}
\end{array}\right)
$$

for $1 \leq i \leq k$, where $x_{i j} \otimes y_{i j}=z_{i j}$. Let $S=K \times Q^{*}$ and define a collection of $3 k$-tricycles $T$ of $K_{k t, k t, k t}$ based on $S \times\{1,2,3\}$ as follows:

(1) If $\left(\left(x_{1}, 1\right),\left(y_{1}, 2\right),\left(z_{1}, 3\right), \ldots,\left(z_{k}, 3\right)\right) \in P$, place the $k^{2} 3 k$-tricycles

$$
\begin{aligned}
& \left(\left(a x_{1}, 1\right),\left(b y_{1}, 2\right),\left(c z_{1}, 3\right),\left(a x_{2}, 1\right),\left(b y_{2}, 2\right),\left(c z_{2}, 3\right), \ldots,\left(a x_{k}, 1\right),\left(b y_{k}, 2\right),\right. \\
& \left.\left(c z_{k}, 3\right)\right)
\end{aligned}
$$

in $T$, for all $a, b \in K$ and $a \otimes b=c$.

(2) If $((a, 1),(b, 2))$ does not belong to a $3 k$-tricycle of $P$, place the $3 k$-tricycle

$$
\begin{aligned}
& \left(\left(a x_{i 1}, 1\right),\left(b y_{i 1}, 2\right),\left(c z_{i 1}, 3\right),\left(a x_{i 2}, 1\right),\left(b y_{i 2}, 2\right),\left(c z_{i 2}, 3\right), \ldots,\left(a x_{i k}, 1\right),\right. \\
& \left.\left(b y_{i k}, 2\right),\left(c z_{i k}, 3\right)\right)
\end{aligned}
$$

in $T$ for each transversal $\pi_{i}$ of $(Q, \otimes)$ where $a \circ b=c$ in the partial quasigroup $\left(Q^{*}, \circ\right)$. (This gives a total of $k 3 k$-tricycles for each such $((a, 1),(b, 2))$.)

ClAIM. $(S \times\{1,2,3\}, T)$ is a $3 k T S$. The proof consists of showing that $|T|=k t^{2}$ and that every edge belongs to at least one $3 k$-tricycle of $T$. The first part is easy. Let $|P|=x$, then $T$ contains $x k^{2}$ type (1) $3 k$-tricycles. Since each $3 k$-tricycle defines $k$ products in the embedding pair $\left(Q, \circ_{1}\right)$ and $\left(Q, \circ_{2}\right)$, there are $k\left(t^{2}-x k\right)$ type (2) $3 k$-tricycles. Hence $|T|=x k^{2}+k t^{2}-x k^{2}=k t^{2}$.

We now show that every edge belongs to at least one $3 k$-tricycle of $T$. There are three cases to consider: (1) $\left(\left(k_{1} x, 1\right),\left(k_{2} y, 2\right)\right) ;(2)\left(\left(k_{1} x, 1\right),\left(k_{3} z, 3\right)\right) ;$ and (3) $\left(\left(k_{2} y, 2\right),\left(k_{3} z, 3\right)\right)$. We will handle each case in turn.

(1) $\left(\left(k_{1} x, 1\right),\left(k_{2} y, 2\right)\right)$. If $((x, 1),(y, 2))$ belongs to a $3 k$-tricycle of $P$, then $\left(\left(k_{1} x, 1\right),\left(k_{2} y, 2\right)\right)$ belongs to a $3 k$-tricycle of type $(1)$. If $((x, 1),(y, 2))$ does not belong to a $3 k$-tricycle of $P$, then $x \circ y=z$ is computed in the partial quasigroup $\left(Q^{*}, \circ\right)$, and so $\left(\left(k_{1} x, 1\right),\left(k_{2} y, 2\right)\right)$ belongs to the $3 k$-tricycle $\left(\ldots,\left(x_{i j} x, 1\right),\left(y_{i j} y, 2\right),\left(z_{i j} z, 3\right)\right.$, $\ldots)$ of type (2), where $k_{1}=x_{i j}$ and $k_{2}=y_{i j}$.

(2) $\left(\left(k_{1} x, 1\right),\left(k_{3} z, 3\right)\right)$. If $((x, 1),(z, 3))$ belongs to a $3 k$-tricycle of $P$, then $\left(\left(k_{1} x, 1\right),\left(k_{3} z, 3\right)\right)$ belongs to a $3 k$-tricycle of type $(1)$. If $((x, 1),(z, 3))$ does not belong to a $3 k$-tricycle of $P$, then there is a unique $y$ such that $x \circ y=z$ in 
the partial quasigroup $\left(Q^{*}, 0\right)$. Moreover, there is a unique transversal such that $x_{i j}=k_{1}$ and $z_{i(j-1)}=k_{3}$, and so $\left(\left(k_{1} x, 1\right),\left(k_{3} z, 3\right)\right)$ belongs to the $3 k$-tricycle $\left(\ldots,\left(z_{i(j-1)} z, 3\right),\left(x_{i j} x, 1\right),\left(y_{i j} y, 2\right),\left(z_{i j} z, 3\right), \ldots\right)$ of type $(2)$, where $k_{1}=x_{i j}$ and $k_{3}=z_{i(j-1)}$.

(3) $\left(\left(\boldsymbol{k}_{2} \boldsymbol{y}, 2\right),\left(\boldsymbol{k}_{3} z, 3\right)\right)$. If $((y, 2),(z, 3))$ belongs to a $3 k$-tricycle of $P$, then $\left(\left(k_{2} y, 2\right)\right.$, $\left.\left(k_{3} z, 3\right)\right)$ belongs to a $3 k$-tricycle of type (1). Otherwise, $x \circ y=z$ for a unique $x$ in the partial quasigroup $\left(Q^{*}, o\right)$. Hence $\left(\left(k_{2} y, 2\right),\left(k_{3} z, 3\right)\right)$ belongs to the $3 k$-tricycle $\left(\ldots,\left(x_{i j} x, 1\right),\left(y_{i j} y, 2\right),\left(z_{i j} z, 3\right), \ldots\right)$ of type (2), where $k_{2}=y_{i j}$ and $k_{3}=z_{i j}$.

Combining cases (1), (2), and (3) shows that $(S \times\{1,2,3\}, T)$ is a $3 k T S$. Since $(K, \otimes)$ is idempotent, the type (1) $3 k$-tricycles produce $k$ disjoint copies of $(Q \times$ $\{1,2,3\}, P)$ in $(S \times\{1,2,3\}, T)$.

Second case: $3 k \in\{6,18\}$. Clearly the proof here is a little different, since there does not exist a pair of orthogonal latin squares of order 2 or of order 6 , but the construction is the same in principle. As in the previous case, from the partial $3 k T S$ $(Q \times\{1,2,3\}, P)$ of order $n$ we define the partial groupoids $\left(Q, \circ_{1}\right)$ and $\left(Q, \circ_{2}\right)$, and let $\left(Q^{*}, \circ\right)$ be a partial quasigroup of order $t \geq 2 n$ into which this embedding pair can be embedded. Let $(K, \otimes)$ be an idempotent quasigroup of order $2 k$. Finally, we need some half-transversals: $n / 2$ cells in a latin square of order $n$, no two in the same row or column, and no two containing the same symbol. When $3 k=6$ we use the following quasigroup $\left(L_{4}, \odot_{4}\right)$ of order $2 k=4$ :

\begin{tabular}{c|c|c|c|c|}
$\odot_{4}$ & \multicolumn{1}{|c}{1} & \multicolumn{2}{c}{2} & \multicolumn{1}{c}{4} \\
\hline 1 & 1 & 2 & 3 & 4 \\
\cline { 2 - 5 } 2 & 3 & 4 & 1 & 2 \\
\cline { 2 - 5 } 3 & 2 & 1 & 4 & 3 \\
\cline { 2 - 5 } 4 & 4 & 3 & 2 & 1 \\
\cline { 2 - 5 } & & &
\end{tabular}

The first $k=2$ rows of $\left(L_{4}, \odot_{4}\right)$ can be partitioned into $2 k=4$ half-transversals

$$
\pi_{1}=\left(\begin{array}{ll}
1 & 2 \\
1 & 2 \\
1 & 4
\end{array}\right), \quad \pi_{2}=\left(\begin{array}{ll}
1 & 2 \\
2 & 1 \\
2 & 3
\end{array}\right), \quad \pi_{3}=\left(\begin{array}{ll}
1 & 2 \\
3 & 4 \\
3 & 2
\end{array}\right) \quad \text { and } \quad \pi_{4}=\left(\begin{array}{ll}
1 & 2 \\
4 & 3 \\
4 & 1
\end{array}\right),
$$

and similarly the last $k=2$ rows of $\left(L_{4}, \odot_{4}\right)$ can be partitioned into $2 k=4$ halftransversals

$$
\pi_{i}=\left(\begin{array}{cc}
x_{i 1}=k+1 & x_{i 2}=k+2 \\
y_{i 1} & y_{i 2} \\
z_{i 1} & z_{i 2}
\end{array}\right), \quad 2 k+1=5 \leq i \leq 8=4 k .
$$


These will be used in the case $3 k=6$. When $3 k=18$ we use the quasigroup ( $\left.L_{12}, \odot_{12}\right)$ of order $2 k=12$ which is the direct product of $\left(L_{4}, \odot_{4}\right)$ with a quasigroup of order 3 . Then clearly the first $k=6$ rows of $\left(L_{12}, \odot_{12}\right)$ can be partitioned into $2 k$ half-transversals

$$
\pi_{i}=\left(\begin{array}{cccc}
x_{i 1}=1 & x_{i 2}=2 & \cdots & x_{i k}=6=k \\
y_{i 1} & y_{i 2} & \ldots & y_{i k} \\
z_{i 1} & z_{i 2} & \cdots & z_{i k}
\end{array}\right), \quad 1 \leq i \leq 12=2 k
$$

and the last $k=6$ rows of $\left(L_{12}, \odot_{12}\right)$ can be partitioned into $2 k$ half-transversals

$$
\pi_{i}=\left(\begin{array}{cccc}
x_{i 1}=k+1 & x_{i 2}=k+2 & \cdots & x_{i k}=2 k \\
y_{i 1} & y_{i 2} & \cdots & y_{i k} \\
z_{i 1} & z_{i 2} & \cdots & z_{i k}
\end{array}\right), \quad 2 k+1 \leq i \leq 4 k .
$$

For $3 k \in\{6,18\}$ we can now define $S=L_{2 k} \times Q^{*}$ and define a collection of $3 k$-tricycles $T$ of $K_{2 k t, 2 k t, 2 k t}$ based on $S \times\{1,2,3\}$ as follows.

(1) If $\left(\left(x_{1}, 1\right),\left(y_{1}, 2\right),\left(z_{1}, 3\right), \ldots,\left(z_{k}, 3\right)\right) \in P$, place the $(2 k)^{2} 3 k$-tricycles

$$
\begin{aligned}
& \left(\left(a x_{1}, 1\right),\left(b y_{1}, 2\right),\left(c z_{1}, 3\right),\left(a x_{2}, 1\right),\left(b y_{2}, 2\right),\left(c z_{2}, 3\right), \ldots\left(a x_{k}, 1\right),\left(b y_{k}, 2\right),\right. \\
& \left.\left(c z_{k}, 3\right)\right)
\end{aligned}
$$

in $T$, for all $a, b \in K$ and $a \otimes b=c$.

(2) If $((a, 1),(b, 2))$ does not belong to a $3 k$-tricycle of $P$, place the $3 k$-tricycle

$$
\begin{aligned}
& \left(\left(a x_{i 1}, 1\right),\left(b y_{i 1}, 2\right),\left(c z_{i 1}, 3\right),\left(a x_{i 2}, 1\right),\left(b y_{i 2}, 2\right),\left(c z_{i 2}, 3\right), \ldots,\left(a x_{i k}, 1\right),\right. \\
& \left.\left(b y_{i k}, 2\right),\left(c z_{i k}, 3\right)\right)
\end{aligned}
$$

in $T$ for each half-transversal $\pi_{i}$ of $\left(L_{k}, \odot_{k}\right), 1 \leq i \leq 4 k$, where $a \circ b=c$ in $\left(Q^{*}, \circ\right)$. (This defines a total of $4 k 3 k$-cycles for each such $((a, 1),(b, 2))$.) The proof these $4 k t^{2} 3 k$-tricycles in $(S \times\{1,2,3\}, T)$ form a $3 k T S$ is virtually identical to the proof given earlier in the case where $3 k \notin\{6,18\}$.

Putting all this together gives the following result.

THEOREM 3.1. A partial $3 k T S$ of order $n$ can be embedded in a $3 k T S$ of order $k t$ if $k \notin\{2,6\}$ and of order $2 k t$ if $k \in\{2,6\}$, for any $t \geq 2 n$.

\section{Final comments}

In the introduction, we described the well-known equivalence between quasigroups of order $n$ and 3-tricycle systems of $K_{n, n, n}$. In Section 2, the patterned hole 
$\left(\left(Q, \circ_{1}\right),\left(Q, \circ_{2}\right),(P, \circ)\right)$ was defined. Patterned holes were introduced in [2] where they provided the means of obtaining small embeddings of partial cycle systems of $K_{n}$, the complete graph. Our final remark is to point out that patterned holes are generalizations of quasigroups, and that this generalization can easily be seen by noticing a correspondence between patterned holes and tricycle systems: A tricycle system of order $n$ is a pair $(Q \times\{1,2,3\}, T)$, where $T$ is a collection of edge-disjoint tricycles (of possibly different lengths), which partition $K_{n, n, n}$ based on $Q \times\{1,2,3\}$. So the difference between a $3 k$-tricycle system and a tricycle system is that in a tricycle system not all tricycles need to have the same length $3 k$.

Given a tricycle system $(Q \times\{1,2,3\}, T)$ of order $n$, we construct a patterned hole $\left(\left(Q, \circ_{1}\right),\left(Q, \circ_{2}\right),(P, \circ)\right)$ as follows. For each $3 k$-tricycle $\left(\left(x_{1}, 1\right),\left(y_{1}, 2\right),\left(z_{1}, 3\right), \ldots\right.$, $\left.\left(z_{k}, 3\right)\right)$ in $T$ with $k>1$, for $1 \leq i \leq k$ define $x_{i} \circ_{1} y_{i}=z_{i-1}$ in $\left(Q, \circ_{1}\right)$ (reducing the subscript modulo $k$ ), and define $x_{i} \circ_{2} y_{i}=z_{i}$ in $\left(Q, o_{2}\right)$. For each 3-tricycle $((x, 1),(y, 2),(z, 3))$ we get a choice: either define $x \circ y=z$ in $(P, \circ)$; or define $x \circ_{1} y=z$ in $\left(Q, \circ_{1}\right)$ and define $x \circ_{2} y=z$ in $\left(Q, \circ_{2}\right)$. It is easy to see that this defines a patterned hole.

EXAMPLE 4.1. To understand the choice offered for each 3-tricycle, consider the following. The patterned hole $\left(\left(Q, \circ_{1}\right),\left(Q, \circ_{2}\right),(P, \circ)\right)$ defined in Examples 2.1 and 2.2 results from a tricycle system that includes the 3 -tricycle $((2,1),(6,2),(4,3))$. In this case, this 3-tricycle was used to define $2 \circ_{1} 6=4$ and $2 \circ_{2} 6=4$. However, note that a patterned hole would still result if we defined $2 \circ 6=4$ in $(P, 0)$ instead, leaving $2 \circ_{1} 6$ and $2 \circ_{2} 6$ undefined.

Conversely, suppose that we are given a patterned hole $\left(\left(Q, \circ_{1}\right),\left(Q, \circ_{2}\right),(P, \circ)\right)$, of order $n$. We define a tricycle system of $K_{n, n, n}$ as follows. For each product $x \circ y=z$ defined in $(P, \circ)$, define the 3-tricycle $((x, 1),(y, 2),(z, 3))$. Let $G$ be the graph formed from $K_{n, n, n}$ by removing the edges $\{(x, 1),(y, 2)\},\{(y, 2),(z, 3)\}$ and $\{(x, 1),(z, 3)\}$ for each $x \circ y=z$ defined in $(P, 0)$. Then there exists a tricycle system of $G$, as the following shows. Form a directed graph $D$ from $G$ by replacing the edge $\{(x, a),(y, a+1)\}$ with the arc directed from $(x, a)$ to $(y, a+1)$ (reducing the second component modulo 3 ). For each vertex $v$ in $D, v$ has the same indegree as its outdegree, so $D$ has a directed Euler tour. This directed Euler tour can be expressed as the union of arc-disjoint directed cycles, and the underlying simple graph of each such directed cycle is clearly a tricycle in $G$. Therefore there exists a tricycle system of $G$.

In fact there may exist many tricycle systems of $G$, so it is natural to ask which patterned holes are defined from the resulting tricycle systems of $K_{n, n, n}$. Notice that for each patterned hole $\left(\left(Q, \circ_{1}\right),\left(Q, \circ_{2}\right),(P, \circ)\right)$, we can define closely related patterned holes $\left(\left(Q, *_{1}\right),\left(Q, *_{2}\right),(P, 0)\right)$ by performing the operations: 
(1) rearrange the symbols among the filled cells within each row of $\left(Q, \circ_{1}\right)$ to form $\left(Q, *_{1}\right)$, and

(2) rearrange the symbols among the filled cells within each column of $\left(Q, \mathrm{O}_{2}\right)$ to form $\left(Q, *_{2}\right)$.

It is easy to see that the patterned holes formed from $\left(\left(Q, \circ_{1}\right),\left(Q, \circ_{2}\right),(P, \circ)\right)$ by using these operations are precisely the patterned holes that are formed from the tricycle systems of $G$, together with the 3 -tricycles used to define $(P, \circ)$.

\section{References}

[1] T. Evans, 'Embedding incomplete latin squares', Amer. Math. Monthly 67 (1960), 958-961.

[2] C. C. Lindner and C. A. Rodger, 'Generalized embedding theorems for partial latin squares', Bull. ICA 5 (1992), 81-99.

[3] — , 'A partial $m=(2 k+1)$-cycle system of order $n$ can be embedded in an $m$-cycle system of order $(2 n+1) m$ ', Discrete Math. 117 (1993), 151-159.

Department of Discrete and Statistical Sciences

120 Mathematics Annex

Auburn University

Alabama 36849-5307

USA 